\title{
TPH2 polymorphisms and expression in Prader-Willi syndrome subjects with differing genetic subtypes
}

\author{
Rebecca S. Henkhaus • Douglas C. Bittel • \\ Merlin G. Butler
}

Received: 23 December 2009/Accepted: 7 May 2010/Published online: 21 May 2010

(C) Springer Science+Business Media, LLC 2010

\begin{abstract}
Prader-Willi syndrome (PWS) is a genetic imprinting disease that causes developmental and behavioral disturbances resulting from loss of expression of genes from the paternal chromosome 15q11-q13 region. In about $70 \%$ of subjects, this portion of the paternal chromosome is deleted, while $25 \%$ have two copies of the maternal chromosome 15, or uniparental maternal disomy (UPD; the remaining subjects have imprinting center defects. There are several documented physical and behavioral differences between the two major PWS genetic subtypes (deletion and UPD) indicating the genetic subtype plays a role in clinical presentation. Serotonin is known to be disturbed in PWS and affects both eating behavior and compulsion, which are reported to be abnormal in PWS. We investigated the tryptophan hydroxylase gene (TPH2), the rate-limiting enzyme in the production of brain serotonin, by analyzing three different $T P H 2$ gene polymorphisms, transcript expression, and correlation with PWS genetic subtype. DNA and RNA from lymphoblastoid cell lines derived from 12 PWS and 12 comparison subjects
\end{abstract}

R. S. Henkhaus $\cdot$ M. G. Butler

Departments of Psychiatry \& Behavioral Sciences and Pediatrics, University of Kansas Medical Center,

3901 Rainbow Blvd.,

Kansas City, KS 66160, USA

D. C. Bittel

Department of Pediatrics, Children's Mercy Hospitals and Clinics, University of Missouri-Kansas City School of Medicine,

2401 Gillham Rd.,

Kansas City, MO 64108, USA

M. G. Butler $(\bowtie)$

Psychiatry \& Behavioral Sciences and Pediatrics,

MS 4015, 3901 Rainbow Blvd.,

Kansas City, KS 66160, USA

e-mail: mbutler4@kumc.edu were used for the determination of genetic subtype, TPH2 polymorphisms and quantitative RT-PCR analysis. A similar frequency of TPH2 polymorphisms was seen in the PWS and comparison subjects with PWS deletion subjects showing increased expression with one or more TPH2 polymorphism. Both PWS deletion and PWS UPD subjects had significantly lower TPH2 expression than control subjects and PWS deletion subjects had significantly lower TPH2 expression compared with PWS UPD subjects. PWS subjects with 15q11-q13 deletions had lower TPH2 expression compared with PWS UPD or control subjects, requiring replication and further studies to identify the cause including identification of disturbed gene interactions resulting from the deletion process.

Keywords Prader-Willi syndrome $\cdot T P H 2$ polymorphisms and expression $\cdot$ Serotonin $\cdot$ Genetic subtypes

\section{Introduction}

Prader-Willi syndrome (PWS) is a genetic imprinting disorder affecting about one in 20,000 live births (Butler et al. 2006). PWS is caused by a disturbance in the paternally-derived chromosome 15q11-q13 region (Bittel and Butler 2005). In about $70 \%$ of subjects, this disturbance results from a 15q11-q13 deletion, while $25 \%$ have maternal uniparental disomy (UPD) due to both copies of chromosome 15 inherited from the mother and the remaining subjects have imprinting defects (Bittel and Butler 2005). Individuals with PWS present with infantile hypotonia, hypogonadism, poor suck and feeding difficulties, high pain threshold, compulsive tendencies and other behavioral problems, mental deficiency for family background, short stature, small hands and feet, and perhaps the 
most defining feature of rapid weight gain in early childhood and obesity due to hyperphagia and inability to experience satiety (Holsen et al. 2009). PWS subjects with the 15q11-q13 deletion and those with UPD have distinct clinical differences (Butler et al. 2004; Cassidy et al. 1997; Dimitropoulos et al. 2000; Holsen et al. 2009). Overall, PWS subjects with 15q11-q13 deletions have more "typical" PWS facial features, lower verbal IQ scores, a higher incidence of obsessive-compulsive (OCD) behaviors, skin-picking, and hypopigmentation (Butler et al. 2006; Cassidy et al. 1997; Dimitropoulos et al. 2000). PWS subjects with UPD tend to have higher incidences of psychosis and social impairment, lower daily living scores, and lower skill levels at tasks requiring spatial skills, like jigsaw puzzles (Dykens and Shah 2003; Zarcone et al. 2007). The two groups have comparable degrees of hyperphagia, and other PWS-associated physical traits such as small hands and feet, hypoplastic genitalia and neonatal hypotonia (Butler et al. 2006).

Perturbations in serotonin biology could account for several of the behavioral problems seen in PWS subjects. Serotonin is a neurotransmitter that plays a role in diverse mental functions including mood, anxiety, alcohol and drug dependency, food intake, sexual behavior, sleep regulation and OCD (Veenstra-VanderWeele et al. 2000). Elevated levels of serotonin have been observed in whole blood samples from autistic subjects while significantly low levels of the serotonin metabolite, 5-HIAA, were found in the cerebrospinal fluid (CSF) of subjects with personality disorders and those who committed or attempted suicide due to unipolar depression (Veenstra-VanderWeele et al. 2000). Altered serotonin signaling in PWS subjects could be caused by either abnormal expression of serotonin and/ or its receptors (e.g., 5HT2C) or altered serotonin metabolism. Interestingly, the 15q11-q13 region includes sequences for several non-coding RNAs that are normally expressed only from the paternal chromosome including 47 nearly identical copies of the snoRNA HBII-52 (SNORD115) which influence expression of 5HT2C serotonin receptors (Kishore and Stamm 2006). The snoRNA HBII-52 promotes alternative splicing of 5HT2C, and without the influence of HBII-52, a decrease in functional 5HT2C is present in the brain of PWS subjects. Further evidence of disturbed serotonin biology in PWS includes significantly higher levels of 5-HIAA in the CSF of PWS subjects, indicating an elevated rate of metabolism and serotonin turnover (Akefeldt et al. 1998). In addition, PWS subjects generally respond positively to selective serotonin reuptake inhibitors (SSRIs) with decreases in severity of skin picking, OCD and aggressive tendencies (Dykens and Shah 2003).

One mechanism for disturbance in serotonin signaling is at the level of serotonin production from tryptophan (Fig. 1). This neurotransmitter is produced by one of two isoforms of tryptophan hydroxylase, TPH1 or TPH2. TPH1 is well-characterized and expressed primarily in the pineal gland and in the gut, while TPH2 is expressed predominantly in the brain and has only recently been described (Chen et al. 2008). Since its discovery, several gene polymorphisms have been characterized. Relevant to our study are three single nucleotide polymorphisms (SNPs) in the 5'UTR of $T P H 2$, two of which are in the promoter, $703 \mathrm{G} / \mathrm{T}$ and $-473 \mathrm{~T} / \mathrm{A}$, with the other SNP located in the 5'UTR of exon $1,+90 \mathrm{~A} / \mathrm{G}$. Studies performed using in vitro models have shown that these SNPs can affect $T P H 2$ gene expression. For example, in primary serotonergic neurons, the $-473 \mathrm{~T} / \mathrm{A}$ polymorphism causes decreased transcription from the $\mathrm{TPH} 2$ promoter, while a polymorphism at the $+90 \mathrm{~A} / \mathrm{G}$ site causes increased expression (Chen et al. 2008; Scheuch et al. 2007). The purpose of the current study is to examine the effect of these SNPs on TPH2 gene expression levels in PWS and control subjects and to determine if there are relevant differences in TPH2 expression between the deletion and UPD genetic subtypes of PWS.

\section{Materials and methods}

Subjects and genetic analysis After informed consent, 37 Caucasian PWS and 12 comparison subjects ranging in age from 10-50 years were studied. Lymphoblastoid cell lines were established via EBV infection from PWS and cognitive/BMI/aged comparison subjects. PWS genetic subtyping was performed as previously described to determine deletion or UPD status (Bittel et al. 2006). DNA was isolated from the lymphoblastoid cell lines and PCR was performed to amplify an 1,154 bp fragment of the $\mathrm{TPH} 2$ gene that includes all three relevant SNPs using the following primers: forward 5' ACTCTGCATAGAGGCAT
Fig. 1 Normal serotonin biology in the human brain

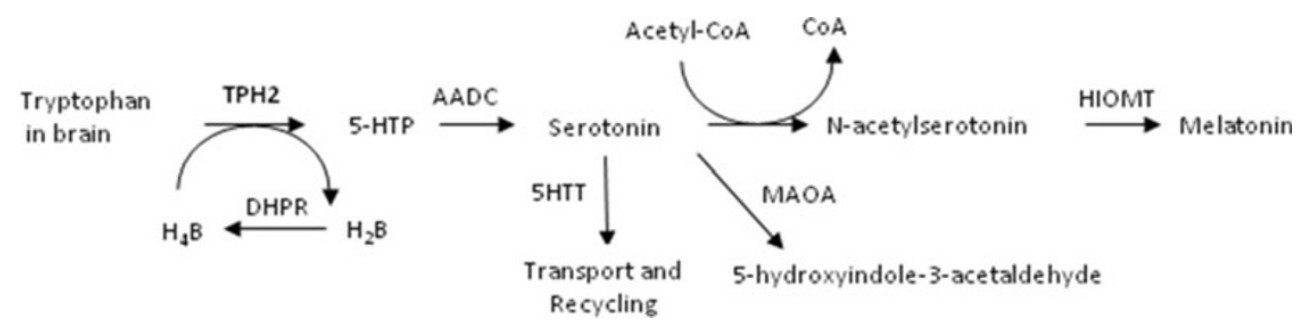


CACAGGA 3'; reverse 5' GGAGAAATTTGAGGTGTGC GTGCT 3'. Sequencing of the fragment was performed using the ABI Genetic Analyzer 3100-Avant (Applied Biosystems, Foster City, CA) and standard protocols.

Gene expression analysis Quantitative real-time PCR (RTPCR) was performed using the QuantiTect SYBR Green One Step RT-PCR kit (Qiagen, Valencia, CA). Briefly, RNA was isolated from actively growing lymphoblastoid cell lines using Trizol reagent (Invitrogen, Carlsbad, CA). RNA (500 ng) was combined with TPH2 or GAPDH (an internal control housekeeping gene) specific primers along with components necessary for both the reverse transcription and the PCR amplification steps as described previously (Bittel et al. 2006). At least three replicates were run for each sample. The reactions were carried out using the ABI 7000 amplification system (Applied Biosystems, Foster City, CA). For 45-50 cycles, the SYBR Green fluorescence was measured during the extension step of each cycle. Data was collected at the point at which the intensity level crosses the cycle threshold $\left(\mathrm{C}_{\mathrm{T}}\right)$, defined as the narrowest point between individual reactions in the logarithmic phase of the reaction. TPH2 gene expression was quantified using the comparative $\mathrm{C}_{\mathrm{T}}$ method. Briefly, the $\Delta \mathrm{C}_{\mathrm{T}}$ was calculated by subtracting the $\mathrm{C}_{\mathrm{T}}$ of the housekeeping gene, GAPDH, from the $T P H 2 \mathrm{C}_{\mathrm{T}}$ value for each individual sample replicate. Then, the $\Delta \Delta \mathrm{C}_{\mathrm{T}}$ value was calculated by subtracting the $\Delta C_{T}$ of a reference DNA sample from each individual sample $\Delta \mathrm{C}_{\mathrm{T}}$ value. Finally, the normalized expression value (RQ) was calculated as $2^{-\left[\Delta \Delta C_{T}\right]}$ as is standard for the comparative $C_{T}$ method. Each sample was run in triplicate and an average was calculated for each sample.

\section{Results}

Thirty-seven PWS subjects were originally analyzed for the status of the TPH2 -703G/T SNP. Of these subjects, six PWS subjects with UPD and six PWS subjects with 15q11-q13 deletions exhibiting either wild-type or differences in the $-703 \mathrm{G} / \mathrm{T}$ SNP were selected for further analysis along with twelve comparison subjects. Subjects were analyzed for three SNPs $(-703 \mathrm{G} / \mathrm{T},-473 \mathrm{~T} / \mathrm{A}$, and $+90 \mathrm{~A} / \mathrm{G})$ in the $T P H 2$ gene, as well as TPH2 transcript expression (Fig. 2). Of the twenty-four subjects selected for complete gene analysis, $15 / 24$ or $62.5 \%$ were wild type for all 3 SNPs. There were subjects who were heterozygous for one, two or three of the SNPs, and one subject was homozygous for all three SNPs. No significant difference was seen between PWS and comparison subjects, or between PWS genetic

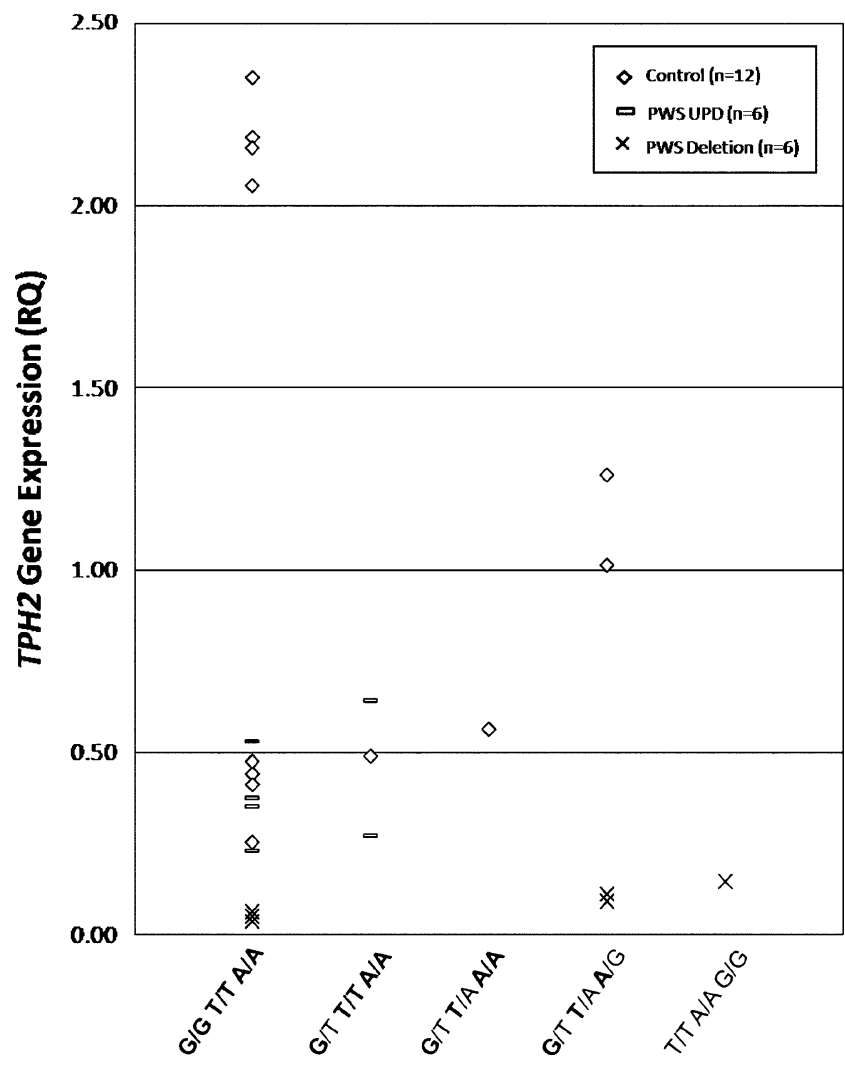

Fig. 2 Quantitative RT-PCR analysis of TPH2 mRNA level in PWS 15q11-q13 deletion subjects $(n=6)$, PWS maternal disomy (UPD) subjects $(n=6)$, and control subjects $(n=12)$. PWS deletion subjects all showed lower TPH2 expression compared with PWS UPD or control subjects. $R Q$ is the normalized expression value $\left(R Q=2^{-\left[\Delta \Delta C_{T}\right]}\right)$, and higher RQ values represent increased gene expression. TPH2 expression values were normalized to GAPDH (see Materials and Methods section for details). Data are stratified by the genotype at three separate SNP loci: $-703 \mathbf{G} / \mathrm{T} ;-473 \mathbf{T} / \mathrm{A} ;+90 \mathrm{~A} / \mathrm{G}$ (wild type alleles are in bold-face type)

subtypes regarding the frequency of SNPs in the TPH2 gene.

A significant difference was observed between control, PWS UPD and PWS 15q11-q13 deletion subjects in the mRNA (transcript) expression of TPH2. The mean RQ values (regardless of SNP status) for the control subjects, PWS UPD and PWS deletion subjects were $1.152+/$ $0.100,0.402+/-0.051$ and $0.046+/-0.015$, respectively (Fig. 2). Compared to control subjects, PWS UPD subjects have a $65 \%$ decrease in TPH2 expression, and PWS deletion subjects have a $96 \%$ decrease in expression in lymphoblastoid cell lines. PWS deletion subjects expressed only $11.5 \%$ of the amount of TPH2 that PWS UPD subjects expressed. Using the student's T-test, the PWS deletion subjects had statistically significantly decreased expression compared to both control subjects $(p<0.0001)$ and PWS UPD subjects $(p<0.0001)$. Overall, PWS UPD subjects also had significantly less TPH2 expression than control subjects $(p<0.0001)$. PWS deletion subjects with TPH 2 
SNPs had significantly increased TPH2 expression compared to subjects with the wild-type THP2 sequence. PWS deletion subjects with no TPH2 polymorphisms (i.e., GG/TT/AA genotypes) had an average RQ value of 0.047 , while PWS deletion subjects with polymorphisms at each $T P H 2$ SNP locus had an average RQ value of 0.114 $(p<0.0001)$.

\section{Discussion}

Since its initial description, several polymorphisms in the TPH2 gene have been described (Chen et al. 2008; Nielsen et al. 2008). Presence of these gene polymorphisms has been linked to conditions ranging from heroin addiction to OCD and depression (Nielsen et al. 2008). The three SNPs investigated in our study have been previously shown to alter $T P H 2$ gene expression. For example, one study highlighted the role of the $-473 \mathrm{~T} / \mathrm{A}$ SNP indicating that it reduced TPH2 transcriptional activity by $22 \%$ in primary serotonergic neurons (Scheuch et al. 2007). A second study demonstrated that the presence of the $+90 \mathrm{~A} / \mathrm{G}$ polymorphism caused a significant increase in expression using a luciferase reporter assay in RN46A cells, a serotonergic rat raphe cell line (Chen et al. 2008). In addition, electrophoretic mobility shift assay (EMSA) analysis revealed that DNA-protein interactions form with the wild-type $+90 \mathrm{~A}$, indicating an inhibitory element since changing this nucleotide to its $G$ polymorphism causes an increase in gene expression (Chen et al. 2008). It is important to note that previous studies have been performed in differing cell lines using TPH2 plasmid constructs, while the goal of our study was to measure endogenous $\mathrm{TPH} 2$ gene expression. Our observation that the $+90 \mathrm{~A} / \mathrm{G}$ SNP showed increased TPH2 expression in PWS deletion subjects is in agreement with the study published by Chen et al. (2008). We observed that PWS deletion subjects with all wild-type SNPs had the lowest overall expression of TPH2, however, no difference was seen in the frequency of TPH2 polymorphisms depending on whether subjects had PWS or not. An important difference observed in our study occurred between the two PWS genetic subtypes (deletion or UPD). PWS subjects with 15q11-q13 deletions had significantly lower $\mathrm{TPH} 2$ transcript expression compared to PWS UPD subjects. Both PWS subtypes exhibited significantly less $T P H 2$ expression than control subjects. Given these results, it is important to note that the $\mathrm{TPH} 2$ gene, located on chromosome 12 , is not within the deleted chromosome $15 \mathrm{q} 11-\mathrm{q} 13$ region in PWS subjects, suggesting that the decreased $T P H 2$ expression must be due to other factors associated with the deletion or deletion process. There are paternally expressed snoRNAs present in the $15 \mathrm{q} 11-\mathrm{q} 13$ region that influence $5 \mathrm{HT} 2 \mathrm{C}$ serotonin receptor post-transcriptional processing as reported by Kishore and Stamm (2006). To the best of our knowledge, a similar snoRNA-TPH2 interaction has not been reported; however, we cannot rule out a possible influence on TPH2 expression. Furthermore, a trans effect involving a disturbed gene network could account for these disturbances. Other non-coding RNAs such as microRNAs not previously identified in the 15q11-q13 region could play a role or result from position effect of other genes near the 15q11-q13 region impacted by the deletion process.

This difference in TPH2 expression between PWS deletion and UPD subjects, if replicated, has important implications in differences in clinical symptoms found in the two genetic subtypes which could impact therapeutic interventions and research. The role of serotonin in the different findings such as compulsions and skin picking in PWS subjects, particularly those with the 15q11-q13 deletion, needs further investigation. These studies should also include investigation of serotonin metabolism in PWS given evidence of elevated 5-HIAA levels in CSF, another finding supporting irregular serotonin biology in PWS subjects. Our study introduces a key physiological difference in serotonin biology between PWS subjects with 15q11-q13 deletions and those with PWS UPD and offers insight into medical treatment and therapeutic practices for these subjects.

Acknowledgements We thank the individuals with PWS and their families for participation in the study and acknowledge Dr. Nataliya Kibiryeva for expert technical assistance, advice and suggestions. The study was partially supported by the NIH Rare Disease grant U54HD061222 and the Prader-Willi Syndrome Association (USA).

\section{References}

Akefeldt A, Ekman R, Gillberg C, Mansson JE. Cerebrospinal fluid monoamines in Prader-Willi syndrome. Biol Psychiatry. 1998:44:1321-8.

Bittel DC, Butler MG. Prader-Willi syndrome: clinical genetics, cytogenetics and molecular biology. Expert Rev Mol Med. 2005;7:1-20.

Bittel DC, Kibiryeva N, Butler MG. Expression of 4 genes between chromosome 15 breakpoints 1 and 2 and behavioral outcomes in Prader-Willi syndrome. Pediatrics. 2006;118:e1276-83.

Butler MG, Bittel DC, Kibiryeva N, Talebizadeh Z, Thompson T. Behavioral differences among subjects with Prader-Willi syndrome and type I or type II deletion and maternal disomy. Pediatrics. 2004;113:565-73.

Butler MG, Lee PDK, Whitman BY. Management of Prader-Willi syndrome. 3rd ed. NY: Springer-Verlag; 2006.

Cassidy SB, Forsythe M, Heeger S, Nicholls RD, Schork N, Benn P, et al. Comparison of phenotype between patients with PraderWilli syndrome due to deletion $15 \mathrm{q}$ and uniparental disomy 15 . Am J Med Genet. 1997;68:433-40. 
Chen GL, Vallender EJ, Miller GM. Functional characterization of the human TPH2 5' regulatory region: untranslated region and polymorphisms modulate gene expression in vitro. Hum Genet. 2008;122:645-57.

Dimitropoulos A, Feurer ID, Roof E, Stone W, Butler MG, Sutcliffe J, et al. Appetitive behavior, compulsivity, and neurochemistry in Prader-Willi syndrome. Ment Retard Dev Disabil Res Rev. 2000; 6:125-30.

Dykens E, Shah B. Psychiatric disorders in Prader-Willi syndrome: epidemiology and management. CNS Drugs. 2003;17:167-78.

Holsen LM, Zarcone JR, Chambers R, Butler MG, Bittel DC, Brooks WM, et al. Genetic subtype differences in neural circuitry of food motivation in Prader-Willi syndrome. Int J Obes (Lond). 2009;33:273-83.

Kishore S, Stamm S. The snoRNA HBII-52 regulates alternative splicing of the serotonin receptor 2C. Science. 2006;311:230-2.
Nielsen DA, Barral S, Proudnikov D, Kellogg S, Ho A, Ott J, et al. TPH2 and TPH1: association of variants and interactions with heroin addiction. Behav Genet. 2008;38:133-50.

Scheuch K, Lautenschlager M, Grohmann M, Stahlberg S, Kirchheiner $\mathrm{J}$, Zill $\mathrm{P}$, et al. Characterization of a functional promoter polymorphism of the human tryptophan hydroxylase 2 gene in serotonergic raphe neurons. Biol Psychiatry. 2007;62:1288-94.

Veenstra-VanderWeele J, Anderson GM, Cook Jr EH. Pharmacogenetics and the serotonin system: initial studies and future directions. Eur J Pharmacol. 2000;410:165-81.

Zarcone J, Napolitano D, Peterson C, Breidbord J, Ferraioli S, CarusoAnderson $\mathrm{M}$, et al. The relationship between compulsive behaviour and academic achievement across the three genetic subtypes of Prader-Willi syndrome. J Intellect Disabil Res. 2007; $51: 478-87$. 